\title{
DISCRETE UNIFORM SUBGROUPS OF SOLVABLE LIE GROUPS
}

\author{
BY \\ LOUIS AUSLANDER(')
}

In [1], we obtained an algebraic characterization of the fundamental groups of compact solvmanifolds (the homogeneous space of a connected solvable Lie group). In the special case of nilmanifolds, it is known (see [4]), that every fundamental group of a compact nilmanifold can be realized as a discrete uniform subgroup of a connected, simply connected nilpotent Lie group. It was pointed out by H. C. Wang [5] that this result is false in the more general case of solvmanifolds. It is the purpose of this paper to characterize those fundamental groups of compact solvmanifolds which can be imbedded as discrete uniform subgroups of connected, simply connected solvable Lie groups. We will adopt for the rest of this paper the convention that the words solvable (nilpotent) Lie group denotes a connected, simply connected solvable (nilpotent) Lie group.

1. Preliminary discussion and definitions. Let $\Gamma$ be a strongly torsion free $S$ group in the sense of $H$. C. Wang; i.e., $\Gamma$ satisfies the diagram

$$
1 \rightarrow D \rightarrow \Gamma \rightarrow Z^{\bullet} \rightarrow 1
$$

where $D$ is a finitely generated torsion free nilpotent group and where $Z^{\circ}$ is the additive group of integers taken $s$ times. Then $[\Gamma, \Gamma] \subset D$ is nilpotent, where [ , ] denotes the commutator subgroup of the group in the bracket. If $G$ is a torsion free, finitely generated nilpotent group we will use $N(G)$ to denote the unique nilpotent Lie group with $G$ as discrete uniform subgroup.

Lemma 1. There exists a unique maximal nilpotent subgroup $M$ of $\Gamma$ which contains $[\Gamma, \Gamma]$.

Proof. Since $[\Gamma, \Gamma]$ is a normal subgroup of $\Gamma$ and every automorphism of $[\Gamma, \Gamma]$ is uniquely extendable to $N([\Gamma, \Gamma])$, the inner automorphisms of $\Gamma$ induce a homomorphism $\chi$ of $\Gamma$ into the automorphism group of $N([\Gamma, \Gamma])$. In $\chi(\Gamma)$ consider the subgroup $\pi$ of elements whose eigenvalues are all 1. Then $\chi^{-1}(\pi)=M$ clearly satisfies the conditions of the lemma.

Clearly $M$ is a characteristic subgroup of $\Gamma$ and torsion free. Let $A_{1}(\Gamma)$ denote the image of $\Gamma$ in the automorphism group of $N(M), A(N(M))$, obtained by forming inner automorphisms of $\Gamma$. Now we may consider $\Gamma / M$. Let $\Gamma^{*} \subset \Gamma$ be the maximum subgroup of $\Gamma$ such that $\Gamma^{*} \supset M$ and $\Gamma^{*} / M$ is

Received by the editors January 6, 1961.

(1) Research supported by N.S.F. Grant 15565 and O.O.R. contract SAR-DA-19-020ORD-5254. 
torsion free. We may apply the construction of $\mathrm{H}$. C. Wang to the group $S=\Gamma^{*} N(M)$ and obtain $S \subset F \cdot T$ where $F$ is maximal nilpotent and $F \supset N(M)$ as normal subgroup. Hence we may form $A_{1}(F) \subset A(N(M))$.

Definition. We will say that a strongly torsion free $S$ group is algebraic if there exists an abelian analytic group of semi-simple elements $T$ in $A(N(M))$ such that

1. $T$ is in the normalizer of $A_{1}(F)$,

2. $A_{1}(\Gamma) \subset A_{1}(F) \cdot T$,

where the dot denotes the semi-direct product.

Our main theorem can now be stated as follows:

Theorem A. A necessary and sufficient condition for $\Gamma$ to be a discrete uniform subgroup of a solvable Lie group is that $\Gamma$ be an algebraic strongly torsion free $S$ group.

The proof of this theorem will be given at the end of this paper after several preliminaries have been completed.

2. Groups of Type I.

Definition. An algebraic strongly torsion free $S$ group will be said to be of Type I provided:

1. $\Gamma / M$ is torsion free, where $M$ is the maximal nilpotent subgroup containing $[\Gamma, \Gamma]$,

2. If $\theta$ is the eigenvalue of any element of $A_{1}(\Gamma)$ then

$$
\theta|\theta|^{-1}=\cos 2 \pi \rho+i \sin 2 \pi \rho,
$$

where $\rho$ is either zero or irrational.

THEOREM 1. An algebraic strongly torsion free $S$ group of Type I is the discrete uniform subgroup of a solvable Lie group $S(\Gamma)$ with the following properties:

1. $N(M)$ is the maximal normal nilpotent analytic subgroup of $S(\Gamma)$.

2. There exist $\gamma_{1}, \cdots, \gamma_{k}$ whose images form a basis for $\Gamma / M$ which lie on one parameter group $S$ in $S(\Gamma)$.

REMARK. Again in the proof of this lemma, as in the very definition of algebraic strongly torsion free $S$ group our debt to the work of $\mathrm{H}$. C. Wang in [5] will be apparent.

Proof. Let $M$ be the maximal nilpotent subgroup of $\Gamma$ and let $S=\Gamma N(M)$. Then as in $\left[5\right.$, p. 15], let $S_{1}=S \times B$. Then under our hypothesis $S_{1}=F \cdot B$, where $F$ is a normal subgroup of unipotent matrices, $B$ is an abelian group of semi-simple elements and $N(M)$ is a normal subgroup of $S_{1}$ with $S_{1} / N(M)$ abelian. By our hypothesis, we may imbed $F$ in $N(F)$ and $B$ in an analytic abelian group $T$ of semi-simple automorphisms of $N(F)$ such that $T$ induces the trivial automorphism in $N(F) / N(M)$. Let $\gamma_{1}, \cdots, \gamma_{k}$ in $\Gamma$ project into a basis of $\Gamma / M$. Consider $\gamma_{i} \in N(F) \cdot T, i=1,2, \cdots, k$. Then $\gamma_{i}=n_{i} \cdot t_{i}$, $i=1,2, \cdots, k$, where $n_{i}$ and $t_{i}$ are unique, $n_{i}$ lies in $N(F)$ and $t_{i} \in T$. Hence 
$n_{i}$ lies on a one parameter group $P\left(n_{i}\right)$. Since $t_{i}$ is semi-simple and $t_{i}$ acts trivially on $N(F) / N(M)$ and $P\left(n_{i}\right)$ does not lie on $N(M)$, we may choose $x \in N(M)$ such that $x t_{i} x^{-1}$ commutes with $n_{i}$. Then we may choose $t_{i}$ on a one parameter group $P^{\prime}\left(t_{i}\right)$ which lies in $T$ such that $x P^{\prime}\left(t_{i}\right) x^{-1}$ commutes with $P\left(n_{i}\right)$ for all values of the parameters. Let $P\left(t_{i}\right)=x P^{\prime}\left(t_{i}\right) x^{-1}$. The $P\left(\gamma_{i}\right)$ $=P\left(t_{i}\right) P\left(n_{i}\right)$ is a one parameter group through $\gamma_{i}$. Let $S(\Gamma)$ be the group generated by $N(M)$ and $P\left(\gamma_{1}\right), \cdots, P\left(\gamma_{k}\right)$. It is now trivial to see that $S(\Gamma)$ satisfies the requirements of our theorem.

REMARK. We make no uniqueness assertions for the group $S(\Gamma)$.

THEOREM 2. Let $\Gamma$ be an algebraic strongly torsion free $S$ group of Type I and let $S(\Gamma)$ satisfy the conclusions of Theorem 1 . Further let $\theta$ be an automorphism of $\Gamma N(M)$ which is trivial on $\Gamma N(M) / N(M)$. Then $\theta$ can be uniquely extended to an automorphism $\theta^{*}$ of $S(\Gamma)$.

Proof. Let $G=\Gamma N(M)$. Let $G(1,1 / 2)$ be the group generated by $\gamma_{1}^{1 / 2}$ $\in P\left(\gamma_{1}\right)$ such that $\left(\gamma_{1}^{1 / 2}\right)^{2}=\gamma_{1}$ and $G$. Then $G$ is normal in $G(1,1 / 2)$ and every element of $G(1,1 / 2)$ can be written as a word in $\gamma_{1}^{1 / 2}$ and elements of $G$. Define $\theta^{*}\left(\gamma_{1}^{1 / 2}\right)=\gamma_{1}^{1 / 2} k$, where $k$ satisfies the equation

$$
\operatorname{ad}\left(\gamma_{1}^{-1 / 2}\right)(k) \cdot k=h_{1}
$$

where

$$
\operatorname{ad}\left(\gamma_{1}^{-1 / 2}\right) k=\gamma_{1}^{-1 / 2} k \gamma_{1}^{1 / 2}
$$

and

$$
\theta\left(\gamma_{1}\right)=\gamma_{1} h_{1}
$$

By the eigenvalue properties of $\operatorname{ad}\left(\gamma_{1}^{1 / 2}\right)$ the above equation has one and only one solution. Define

$$
\begin{aligned}
\theta^{*}(g) & =\theta(g), \\
\theta^{*}\left(\gamma_{1}^{1 / 2} g\right) & =\gamma_{1}^{1 / 2} k \theta(g) .
\end{aligned} \quad g \in G,
$$

Verify that

$$
\theta^{*}\left(\gamma_{1}^{-1 / 2} g \gamma_{1}^{1 / 2}\right)=k^{-1} \gamma_{1}^{-1 / 2} \theta(g) \gamma_{1}^{1 / 2} k
$$

Now it is straightforward to verify that this is an automorphism of $G(1,1 / 2)$. Similarly, we can define $\theta^{*}$ on the group $G(1 / 2)$ generated by $\gamma_{1}^{1 / 2}, \cdots, \gamma_{k}^{1 / 2}$ and $N(M)$. By induction, we can define $\theta^{*} G(1 / 2 n)$ where $G(1 / 2 n)$ is generated by $\gamma_{1}^{1 / 2 n}, \cdots, \gamma_{k}^{1 / 2 n}$. Let $\omega$ denote the diadic rationals as a topological group, topologized as a subset of the real line. Then $\theta^{*}$ can clearly be extended to be a continuous automorphism of the topological group $G(\omega)$. But $S(\Gamma)$ is the 
completion of $G(\omega)$ and hence we can define $\theta^{*}$ in $S(\Gamma)$. Since $\theta^{*}$ is uniquely defined in $G(\omega)$ it is unique in $S(\Gamma)$.

THEOREM B. Let $\Gamma$ be an algebraic strongly torsion free $S$ group of Type I and let $S(\Gamma)$ be as constructed in Theorem 1 . Further, let $S$ be a solvable Lie group containing $\Gamma$ as a discrete uniform subgroup. Then there exists a compact, connected abelian Lie group of automorphisms $T^{*}$ of $S(\Gamma)$ such that $S \subset S(\Gamma) \cdot T^{*}$, where the dot denotes the semi-direct product.

Proof. Let $N$ be the maximal nilpotent normal subgroup of $S$ and consider $\Gamma N$. Then there is a natural isomorphism of $\Gamma N$ into $\Gamma N(M)$. We will consider $\Gamma N$ as a subgroup of $\Gamma N(M)$ under this identification. Then every automorphism of $\Gamma N$ is uniquely extendable to $\Gamma N(M)$, since $M$ is characteristic in $\Gamma$. But every element $s \in S$ determines, by inner automorphism, an automorphism of $\Gamma N$. Hence we may consider $S$ as a group of automorphisms of $\Gamma N(M)$. Further, since $S / N$ is abelian, $S$ will act trivially on $\Gamma N(M)$. We may therefore by Theorem 2 consider $S$ as a group of automorphisms of $S(\Gamma)$. Let us form $S(\Gamma) \cdot S$, where the dot denotes the semi-direct product. Let $\Delta$ denote the subgroup of $S(\Gamma) \cdot S$ consisting of elements $\left(x, x^{-1}\right)$ for $x \in \Gamma N$. Since $\Gamma N \supset[S(\Gamma), S(\Gamma)]$ it is normal in $S(\Gamma)$ and $\Delta$ is normal in $S(\Gamma) \cdot S$. Further $S$ and $S(\Gamma)$ have isomorphic images in $S(\Gamma) \cdot S / \Delta$. Now the image $G$ of $S(\Gamma)$ is normal in $S(\Gamma) \cdot S / \Delta$ and $S(\Gamma) \cdot S / \Delta / G$ is isomorphic to $S / \Gamma N$ and hence is a compact abelian Lie group. Since any extension of a connected, simply connected solvable Lie group by a compact group is a split extension, we have that $S(\Gamma) \cdot T^{*} \supset S$, where $T^{*}$ is a compact abelian Lie group of automorphisms of $S(\Gamma)$.

\section{Proof of main theorem.}

Lемма 2. Every strongly torsion free $S$ group $\Gamma$ has a characteristic subgroup of finite index $\Gamma^{*}$ such that $\Gamma^{*}$ is an algebraic strongly torsion free $S$ group of Type I and $\Gamma^{*} \supset M$.

The proof of this lemma is immediate and will be omitted.

TheORem A. A necessary and sufficient condition for $\Gamma$ to be a discrete uniform subgroup of a solvable Lie group is that $\Gamma$ be an algebraic strongly torsion free $S$ group.

Proof. Assume that $\Gamma$ is an algebraic strongly torsion free $S$ group. Then let $\Gamma^{*} \subset \Gamma$ satisfy the conclusions of Lemma 2 . Then we may consider $\Gamma$ acting on $N(M)$. Hence we have $\Gamma / \Gamma^{*}$ induces a finite group of automorphisms of $\Gamma^{*} N(M)$ which is trivial on $\Gamma^{*} N(M) / N(M)$. Hence $\Gamma / \Gamma^{*}$ induces a finite group of automorphisms of $S(\Gamma)$. Since it is trivial on $\Gamma^{*} N(M) / N(M)$ and lies on a torus group $T$ of automorphisms of $N(M)$, we can extend this to a torus group of automorphisms of $S(\Gamma)$. Hence $\Gamma \subset S(\Gamma) \cdot T$. But by Theorem 1 in [1], this means that $\Gamma$ is a discrete uniform subgroup of a Lie group. 
Now let $\Gamma \subset S$ be a discrete uniform subgroup. Then $S \subset S\left(\Gamma^{*}\right) \cdot T$, where $\Gamma^{*}$ is an algebraic strongly torsion free $S$ group of Type I. Since $\Gamma \subset S\left(\Gamma^{*}\right) \cdot T$ and $N(M)$ is the maximal analytic nilpotent subgroup, we have that $S\left(\Gamma^{*}\right)$ $\cdot T / N(M)$ is abelian. Hence it is trivial to verify that $\Gamma$ is algebraic.

\section{REFERENCES}

1. L. Auslander, Fundamental groups of compact solvmanifolds, Amer. J. Math. vol. 82 (1960) pp. 689-697.

2. - Solvable Lie groups acting on nilmanifolds, Amer. J. Math. vol. 82 (1960) pp. 653-660.

3. L. Auslander and M. Auslander, Solvable Lie groups acting on solvmanifolds, in preparation.

4. A. I. Malcev, On a class of homogeneous spaces, Amer. Math. Soc. Translations, Series 1, no. 39, 1951. 1-19.

5. H. C. Wang, Discrete subgroups of solvable Lie groups, Ann. of Math. vol. 64 (1956) pp.

YALE UNIVERSITY,

New Haven, Connecticut

INDIANA UNIVERSITY,

BLOOMINGTON, INDIANA 